\title{
Analysis of the Vertical Profile of Concentration in Sediment-Laden Flows
}

\author{
Qing-Quan Liu'; An-Ping Shu²; and Vijay P. Singh, F.ASCE ${ }^{3}$
}

\begin{abstract}
From a theoretical investigation of the sediment concentration distribution in sediment-laden flows, a relationship between the profile of sediment concentration and the intensity of vertical fluctuation of particles was established and indirectly verified using experimental data obtained in a vertical rectangular duct flow. It is shown that sediment suspension and the profile of sediment concentration are significantly influenced by the particle fluctuation. This leads to a new explanation for the mechanism of two patterns of sediment concentration profiles.
\end{abstract}

DOI: 10.1061/(ASCE)0733-9399(2007)133:6(601)

CE Database subject headings: Sediment concentration; Turbulent diffusion; Sediment transport.

\section{Introduction}

Particle transport processes have numerous industrial and practical applications, and have therefore received considerable attention. The knowledge of particle concentration profile is critical to modeling sediment transport in rivers. O'Brien (1933) and Rouse (1937) were among the first to analyze the mechanism by which particles are maintained in suspension in turbulent flow. This leads to the diffusion theory. Although this reasoning formed the basis of several models applied in practical projects (Vanoni 1946; Ismail 1952; Montes and Ippen 1973), it has been found to have serious limitations (Itakura and Kishi 1980; Ni and Wang 1987, 1991a; Kaushal et al. 2002). First, the diffusion theory does not provide the dynamic mechanism of sediment particle suspension. Second, the diffusivity coefficient is usually assumed equal to the kinematic eddy viscosity of the clear water turbulent flow, which, in fact, is not completely true. Third, the effect of particles on the flow structure is not accounted for in the simple diffusion theory. Consequently, different diffusivities have been suggested for different flow regions in order to obtain acceptable results (Batchelor 1965; van Rijn 1984).

There are other theories, on the other hand, such as energy theory, mixture theory, similarity theory, and stochastic theory, that have been proposed to derive different expressions for the

\footnotetext{
${ }^{1}$ Professor, Institute of Mechanics, Chinese Academy of Sciences, Beijing 100080, China. E-mail: qqliu@imech.ac.cn

${ }^{2}$ Associate Professor, College of Water Sciences, Beijing Normal Univ., Beijing, China.

${ }^{3}$ Caroline and William N. Lehrer Distinguished Chair in Water Engineering, Professor of Biology and Agricultural Engineering, and Professor of Civil Engineering, Dept. of Biology and Agricultural Engineering, Texas A\&M Univ., College Station, TX 77843-2117. E-mail: vsingh@tamu.edu

Note. Associate Editor: Nikolaos D. Katopodes. Discussion open until November 1, 2007. Separate discussions must be submitted for individual papers. To extend the closing date by one month, a written request must be filed with the ASCE Managing Editor. The manuscript for this paper was submitted for review and possible publication on September 27, 2005; approved on September 19, 2006. This paper is part of the Journal of Engineering Mechanics, Vol. 133, No. 6, June 1, 2007. (CASCE, ISSN 0733-9399/2007/6-601-607/\$25.00.
}

vertical concentration distribution. A systematic comparison of these expressions reveals that the underlying governing equations have nearly the same form as the diffusion equation, with slightly different expressions for sediment diffusivity coefficient (Ni and Wang 1991b). These theories do not overcome the limitations of the diffusion theory. Nielsen $(1991,1995)$ showed that the pure gradient diffusion is an unsatisfactory modeling principle. An alternative approach is one of combining convection and diffusion, thus dealing explicitly with the upward transport of sediment in traveling vortices (Nielsen 1995). Such approaches not only improve predictions of concentration profiles, but also reconcile the concepts of eddy viscosity and sediment diffusivity.

Recent years have witnessed remarkable advances in twophase flow theory and measurement techniques, especially in the use of laser for evaluating the component velocities of the phases (Tsuji and Morikawa 1982; Lu et al. 1988; Nino and Garcia 1996; Liu 1998; Sommerfeld and Huber 1999; Aísa et al. 2002; Liu and Singh 2004). The effect of suspended sediment on turbulent flow and the difference between water flow and suspended sediment motion is now well recognized. The variation of the mean velocity profile in the vertical plane due to the effect of suspension was considered by Dyer and Soulsby (1988), McLean (1992), and Zhou and Ni (1995). In their two-phase flow model, Wang and $\mathrm{Ni}(1990,1991)$ regarded solid particles as analogous to molecules of gas in the kinetic theory of gases and described them by the Boltzmann equation. Then, combining the kinetic theory and the continuum theory, they obtained an explicit particle velocity distribution function and developed a model for sediment concentration profile. Similar results have also been obtained by Zaichik et al. (1997), Hyland et al. (1999), Xu and Zhou (2000), Derevich (2000), Fu et al. (2001), and Fu and Wang (2003) using the twophase flow theory. These investigations have led to an improved understanding of the mechanism of sediment suspension and concentration distribution. Nevertheless, our understanding of the complex processes governing the sediment-turbulent flow interaction is less than complete. For instance, why do particles suspend in water flow? How are particle movements influenced by fluid turbulence as well as particle-particle interactions? What causes different patterns of concentration profile? Further, formulating the underlying mechanisms is essential for simulation of sediment transport. 
The objective of this study is to investigate the mechanisms of particle suspension, analyze the sediment concentration profile in sediment-laden flow using the two-phase theory, and develop an explanation for the mechanism governing two types of sediment concentration profiles.

\section{Mechanism of Sediment Suspension in Water Flows}

Fundamental to modeling sediment transport in rivers is the mechanism governing sediment particle suspension in water flow. A multitude of investigations have discussed the suspension of sediment particles by analyzing forces acting on a single particle. Actually, the motion of sediment particles in water flow is a kind of group motion. Therefore, in the present study we will analyze the suspension of sediment particles from the point of view of the momentum equilibrium of group particle motion. For sediment-laden flow in an open channel with uniform slope $J(J=\tan \theta \approx \sin \theta, \theta=$ slope angle $)$, the equation of motion for sediment particles in the vertical direction ( $y$-direction) can be expressed as

$$
\frac{\partial \alpha_{s} v_{s}}{\partial t}+\frac{\partial\left(\alpha_{s} u_{s} v_{s}\right)}{\partial x}+\frac{\partial\left(\alpha_{s} v_{s} v_{s}\right)}{\partial y}=-\alpha_{s} g_{y}-\frac{\alpha_{s}}{\rho_{s}} \frac{\partial p}{\partial y}+\frac{f_{y}}{\rho_{s}}+\frac{F_{y}}{\rho_{s}}
$$

where $x=$ coordinate along the flow direction; $y=$ coordinate perpendicular to the $x$-direction; $t=$ time; $u_{s}$ and $v_{s}=$ sediment particle velocity components in the $x$ - and $y$-directions, respectively; $\rho_{s}=$ density of sediment particles; $f_{y}=$ flow resistance in the $y$ direction; $\alpha_{s}=$ fractional number of sediment particles by volume; $p=$ pressure of water flow; $F_{y}=$ uplift force of flow acting on sediment particles; and $g_{y}=$ gravity acceleration component in the $y$-direction. Generally, the slope angle $\theta$ is small, so $g_{y}=g \cos \theta \approx g(g=$ gravity acceleration and $\theta=$ slope angle $)$

Taking time averaging of Eq. (1), one obtains

$$
\begin{aligned}
& \frac{\partial}{\partial t}\left(\overline{\alpha_{s} v_{s}}+\overline{\alpha_{s}^{\prime} v_{s}^{\prime}}\right)+\frac{\partial}{\partial x}\left(\overline{\alpha_{s} u_{s} v_{s}}+\overline{\alpha_{s} u_{s}^{\prime} v_{s}^{\prime}}+\overline{u_{s} \alpha_{s}^{\prime} v_{s}^{\prime}}+\overline{v_{s} \alpha_{s}^{\prime} u_{s}^{\prime}}\right. \\
& \left.+\overline{\alpha_{s}^{\prime} u_{s}^{\prime} v_{s}^{\prime}}\right)+\frac{\partial}{\partial y}\left(\overline{\alpha_{s} v_{s} v_{s}}+\overline{\alpha_{s} v_{s}^{\prime} v_{s}^{\prime}}+\overline{v_{s} \alpha_{s}^{\prime} v_{s}^{\prime}}+\overline{v_{s} \alpha_{s}^{\prime} v_{s}^{\prime}}+\overline{\alpha_{s}^{\prime} v_{s}^{\prime} v_{s}^{\prime}}\right) \\
& =-\overline{\alpha_{s}} g-\frac{\overline{\alpha_{s}}}{\rho_{s}} \frac{\partial \bar{p}}{\partial y}+\overline{\frac{f_{y}}{\rho_{s}}}+\frac{\overline{F_{y}}}{\rho_{s}}+\frac{1}{\rho_{s}} \overline{\alpha_{s}^{\prime} \frac{\partial p^{\prime}}{\partial y}}
\end{aligned}
$$

Eq. (2) can be expressed as

$$
\begin{gathered}
\frac{\partial\left(\overline{\alpha_{s} v_{s}}\right)}{\partial t}+\frac{\partial\left(\overline{\alpha_{s} u_{s} v_{s}}\right)}{\partial x}+\frac{\partial\left(\overline{\alpha_{s} v_{s} v_{s}}\right)}{\partial y}+\frac{\partial\left(\overline{v_{s} \alpha_{s}^{\prime} u_{s}^{\prime}}\right)}{\partial x}+\frac{\partial\left(\overline{v_{s} \alpha_{s}^{\prime} v_{s}^{\prime}}\right)}{\partial y} \\
+\frac{\partial\left(\overline{\alpha_{s}^{\prime} v^{\prime}}\right)}{\partial t}+\frac{\partial}{\partial x}\left(\overline{\alpha_{s} u_{s}^{\prime} v_{s}^{\prime}}+\overline{u_{s} \alpha_{s}^{\prime} v_{s}^{\prime}}+\overline{\alpha_{s}^{\prime} u_{s}^{\prime} v_{s}^{\prime}}\right) \\
+\frac{\partial}{\partial y}\left(\overline{\alpha_{s} v_{s} v_{s}}+\overline{\alpha_{s} v_{s}^{\prime} v_{s}^{\prime}}+\overline{v_{s} \alpha_{s}^{\prime} v_{s}^{\prime}}+\overline{\alpha_{s}^{\prime} v_{s}^{\prime} v_{s}^{\prime}}\right) \\
=-\overline{\alpha_{s}} g-\frac{\alpha_{s}}{\rho_{s}} \frac{\partial \bar{p}}{\partial y}+\frac{\overline{f_{y}}}{\rho_{s}}+\frac{\overline{F_{y}}}{\rho_{s}}+\frac{1}{\rho_{s}} \alpha_{s}^{\prime} \frac{\partial p^{\prime}}{\partial y}
\end{gathered}
$$

Eq. (3) can be rewritten as

$$
\begin{aligned}
\overline{v_{s}}[ & \left.\frac{\partial \overline{\alpha_{s}}}{\partial t}+\frac{\partial\left(\overline{\alpha_{s} u_{s}}\right)}{\partial x}+\frac{\partial\left(\overline{\alpha_{s} v_{s}}\right)}{\partial y}+\frac{\partial\left(\overline{\alpha_{s}^{\prime} u_{s}^{\prime}}\right)}{\partial x}+\frac{\partial\left(\overline{\alpha_{s}^{\prime} v_{s}^{\prime}}\right)}{\partial y}\right] \\
& +\overline{\alpha_{s}} \frac{\partial \overline{v_{s}}}{\partial t}+\left(\overline{\alpha_{s} u_{s}}+\overline{\alpha_{s}^{\prime} u_{s}^{\prime}}\right) \frac{\partial \overline{v_{s}}}{\partial x}+\left(\overline{\alpha_{s} v_{s}}+\overline{\alpha_{s}^{\prime} v_{s}^{\prime}}\right) \frac{\partial \overline{v_{s}}}{\partial y} \\
& +\frac{\partial\left(\overline{\alpha_{s}^{\prime} v^{\prime}}\right)}{\partial t}+\frac{\partial}{\partial x}\left(\overline{\alpha_{s} u_{s}^{\prime} v_{s}^{\prime}}+\overline{u_{s} \alpha_{s}^{\prime} v_{s}^{\prime}}+\overline{\alpha_{s}^{\prime} u_{s}^{\prime} v_{s}^{\prime}}\right) \\
& +\frac{\partial}{\partial y}\left(\overline{\alpha_{s} v_{s}^{\prime} v_{s}^{\prime}}+\overline{v_{s} \alpha_{s}^{\prime} v_{s}^{\prime}}+\overline{\alpha_{s}^{\prime} v_{s}^{\prime} v_{s}^{\prime}}\right) \\
& =-\overline{\alpha_{s} g}-\frac{\alpha_{s}}{\rho_{s}} \frac{\partial p}{\partial y}+\frac{\overline{f_{y}}}{\rho_{s}}+\frac{\overline{F_{y}}}{\rho_{s}}+\frac{1}{\rho_{s}} \overline{\alpha_{s}^{\prime} \frac{\partial p^{\prime}}{\partial y}}
\end{aligned}
$$

Combining the continuity equation of particles in motion yields

$$
\begin{aligned}
& \overline{\alpha_{s}} \frac{\partial \bar{v}_{s}}{\partial t}+\left(\overline{\alpha_{s} u_{s}}+\overline{\alpha_{s}^{\prime} u_{s}^{\prime}}\right) \frac{\partial \overline{v_{s}}}{\partial x}+\left(\overline{\alpha_{s} v_{s}}+\overline{\alpha_{s}^{\prime} v_{s}^{\prime}}\right) \frac{\partial \overline{v_{s}}}{\partial y} \\
& +\frac{\partial\left(\overline{\alpha_{s}^{\prime} v^{\prime}}\right)}{\partial t}+\frac{\partial}{\partial x}\left(\overline{\alpha_{s} u_{s}^{\prime} v_{s}^{\prime}}+\overline{u_{s} \alpha_{s}^{\prime} v_{s}^{\prime}}+\overline{\alpha_{s}^{\prime} u_{s}^{\prime} v_{s}^{\prime}}\right) \\
& +\frac{\partial}{\partial y}\left(\overline{\alpha_{s} v_{s}^{\prime} v_{s}^{\prime}}+\overline{v_{s} \alpha_{s}^{\prime} v_{s}^{\prime}}+\overline{\alpha_{s}^{\prime} v_{s}^{\prime} v_{s}^{\prime}}\right) \\
& =-\overline{\alpha_{s}} g-\frac{\overline{\alpha_{s}}}{\rho_{s}} \frac{\overline{\partial p}}{\partial y}+\frac{\overline{f_{y}}}{\rho_{s}}+\frac{\overline{F_{y}}}{\rho_{s}}+\frac{1}{\rho_{s}} \alpha_{s}^{\prime} \frac{\partial p^{\prime}}{\partial y}
\end{aligned}
$$

For steady uniform channel flow and considering steady equilibrium sediment transport, $\partial / \partial t=\partial / \partial x=0, \partial \overline{v_{s}} / \partial y=0$. Thus Eq. (5) can be simplified as

$$
\begin{aligned}
& \frac{\partial}{\partial y}\left(\overline{\alpha_{s} v_{s}^{\prime} v_{s}^{\prime}}+\overline{v_{s} \alpha_{s}^{\prime} v_{s}^{\prime}}+\overline{\alpha_{s}^{\prime} v_{s}^{\prime} v_{s}^{\prime}}\right) \\
& =-\overline{\alpha_{s}} g-\frac{\overline{\alpha_{s}}}{\rho_{s}} \frac{\partial \bar{p}}{\partial y}+\overline{\frac{f_{y}}{\rho_{s}}}+\frac{\overline{F_{y}}}{\rho_{s}}+\frac{1}{\rho_{s}} \alpha_{s}^{\prime} \frac{\partial p^{\prime}}{\partial y}
\end{aligned}
$$

where $\overline{v_{s} \alpha_{s}^{\prime} v_{s}^{\prime}}=\overline{v_{s} \alpha_{s}} U_{s}^{T} \quad\left(U_{s}^{T}=\right.$ turbulent diffusion velocity of particle). In general, $U_{s}^{T}$ is much less than the velocity fluctuation of particles, $\sqrt{\overline{v_{s}^{\prime 2}}}$. Consequently, neglecting the higher order correlation quantity and other smaller quantities and throwing off the time-averaging symbol, Eq. (6) is finally simplified as

$$
\frac{\partial\left(\alpha_{s} \overline{v_{s}^{\prime} v_{s}^{\prime}}\right)}{\partial y}=-\alpha_{s} g-\frac{\alpha_{s}}{\rho_{s}} \frac{\partial p}{\partial y}+\frac{f_{y}}{\rho_{s}}+\frac{F_{y}}{\rho_{s}}
$$

In addition

$$
\alpha_{s}=\frac{m_{s} n_{s}}{\rho_{s}}, \quad \frac{\partial p}{\partial y}=-\rho g
$$

where $m_{s}=$ mass of sediment particle; $n_{s}=$ number density of sediment particles; $\rho=$ water density, and $\rho_{s}=$ density of sediment particles.

Eq. (7) can be cast in the following form:

$$
\frac{\partial}{\partial y}\left(m_{s} n_{s} \overline{v_{s}^{\prime 2}}\right)=-m_{s} n_{s} g\left(1-\frac{\rho}{\rho_{s}}\right)+f_{y}+F_{y}
$$

The left-hand term of Eq. (9), $\partial\left(m_{s} n_{s} \overline{v_{s}^{\prime 2}}\right) / \partial y$, expresses the rate of momentum transport by sediment particle fluctuations in the vertical plane. It can be thought of as a fluctuating stress caused by the particle fluctuation and is similar to turbulent stress. Liu (1993) analyzed the fluctuation of particles and reasoned that the particle fluctuation can generate partial pressure of the particle 
phase which has significant effect on the movement of particles. Actually, the particle fluctuation may have various forms. Besides heat motion (which can be negligible for sediment particles), and the fluctuation of particles induced by turbulent flow, there is the random motion taking on other forms, such as the saltation of particles and its impact on the bed, etc. (Liu and Lu 1993).

The first term on the right-hand side of Eq. (9), $m_{s} n_{s} g\left(1-\rho / \rho_{s}\right)$ expresses the gravity of particles in water. Eq. (9) shows that the gravity and buoyancy forces acting on sediment particles can be balanced by the momentum of fluctuations of moving particles, the uplift force and the flow resistance that the water flow exerts on the particles. Thus, it can be concluded that the vertical fluctuation of particles is one of the most important mechanisms causing sediment particle suspension and maintaining a steady distribution of sediment concentration in the vertical plane.

\section{Vertical Distribution of Sediment Concentration}

The resistance of flow to particles in the vertical plane is due to the gravity falling of particles. In Eq. (9), the flow resistance $f_{y}$ can be divided into two parts: laminar flow resistance $f_{L}$ and turbulent flow resistance $f_{T}$

$$
f_{y}=f_{L}+f_{T}
$$

The laminar flow resistance can be approximated using Stokes' formula. Many investigations on sediment dynamics (Chien and Wan 1999) have indicated that during the falling of particles the gravity of particles under water is balanced by the laminar flow resistance. This leads to particles falling in water with a constant velocity. Thus

$$
f_{L}=m_{s} n_{s} g\left(1-\frac{\rho}{\rho_{s}}\right)
$$

The turbulent flow resistance is considered to be proportional to the difference of diffusion velocities of two phases, $\Delta U_{y}^{T}$ and the relaxation time is the characteristic time of turbulence, $\tau^{*}$ (Liu 1993). Therefore

$$
f_{T}=\frac{\overline{\sigma \sigma_{s}}}{\bar{\sigma}+\overline{\sigma_{s}}} \frac{\Delta U_{y}^{T}}{\tau^{*}}
$$

where $\sigma=\alpha \rho ; \sigma_{s}=\alpha_{s} \rho_{s}$ ( $\alpha$ and $\alpha_{s}=$ fractional numbers of fluid and sediment particles by volume, respectively, and there is $\left.\alpha+\alpha_{s}=1\right)$. With $\omega$ denoting the velocity of particles falling, one has

$$
\Delta U_{y}^{T}=-\frac{\bar{\sigma}+\overline{\sigma_{s}}}{\bar{\sigma}} \omega
$$

Consequently

$$
f_{T}=-\overline{\sigma_{s}} \frac{\omega}{\tau^{*}}=-m_{s} n_{s} \frac{\omega}{\tau^{*}}
$$

Substituting Eqs. (9), (11), and (14) into Eq. (10) yields

$$
\frac{\partial}{\partial y}\left(m_{s} n_{s} \overline{v_{s}^{\prime 2}}\right)=-m_{s} n_{s} \frac{\omega}{\tau^{*}}+m_{s} n_{s} a
$$

where $a=$ acceleration velocity by the uplift force $F_{y}\left(=m_{s} n_{s} a\right)$. Actually, the term $m_{s} n_{s}$ expresses sediment concentration. Substituting $C$ for $m_{s} n_{s}$ in Eq. (15), one has

$$
\frac{\partial}{\partial y}\left(C \overline{v_{s}^{\prime 2}}\right)=-C \frac{\omega}{\tau^{*}}+C a
$$

or

$$
\frac{\partial}{\partial y}\left[\ln \left(C \overline{v_{s}^{\prime 2}}\right)\right]=-\left(\frac{\omega}{\tau^{*}}+a\right) / \overline{v_{s}^{\prime 2}}
$$

Consequently, the sediment concentration distribution in the vertical plane can be expressed as

$$
C \overline{v_{s}^{\prime 2}}=\left[C \overline{v_{s}^{\prime 2}}\right]_{y=0} \exp \left[-\int_{0}^{y}\left(\frac{\omega}{\tau^{*}}-a\right) / \overline{v_{s}^{\prime 2}} d y\right]
$$

In general, the uplift force can be neglected in the mainstream region of flow, excepting the thin region near the bed. Neglecting the uplift force, the formula for the concentration distribution can be simplified as

$$
C \overline{v_{s}^{\prime 2}}=\left[C \overline{v_{s}^{\prime 2}}\right]_{y=0} \exp \left(-\int_{0}^{y} \frac{\omega}{\tau^{*} \overline{v_{s}^{\prime 2}}} d y\right)
$$

When the sediment particle size is uniform, the profile of the number density of particles can be obtained as

$$
n_{s} \overline{v_{s}^{\prime 2}}=\left[n_{s} \overline{v_{s}^{\prime 2}}\right]_{y=0} \exp \left(-\int_{0}^{y} \frac{\omega}{\tau^{*} \overline{v_{s}^{\prime 2}}} d y\right)
$$

This analysis reveals that the fluctuation stress of particles, i.e., the momentum transformation in the vertical plane caused by particle fluctuation is important for sediment suspension and maintaining a steady profile in water flow.

\section{Experimental Verification}

Eq. (19) cannot be directly verified easily because it includes an unknown quantity $\tau^{*}$ which is difficult to determine. However, this difficulty can be easily overcome in a vertical flow in which the gravity action needs not to be considered. For vertical flow, assuming the $x$-direction expresses the vertical direction (flow direction) and the $y$-direction is the horizontal or transverse direction (perpendicular to the flow direction), the parameter $\omega$ that expresses the falling velocity of particle in the $y$-direction (horizontal direction) is zero. Therefore, neglecting the flow uplift force in the $y$-direction, Eq. (19) can be expressed as

$$
\frac{\partial}{\partial y}\left(C \overline{v_{s}^{\prime 2}}\right)=0
$$

Integration of Eq. (21) leads to

$$
C \overline{v_{s}^{\prime 2}}=K \quad(K=\text { constant })
$$

For uniform particles, one can write

$$
n_{s} \overline{v_{s}^{\prime 2}}=k \quad(k=\text { constant })
$$

Consequently, Eq. (22) or Eq. (23) can be verified using experimental data on vertical flow. Using the laser Doppler split-phase measuring technique, fluctuation velocities of flow and particles, and particle concentration in a vertical rectangular duct flow were observed (Liu and Singh 2004). The experimental setup is shown in Fig. 1. The rectangular duct had a length of $1000 \mathrm{~mm}$, width of $15 \mathrm{~mm}$, and height of $30 \mathrm{~mm}$. The measurement section was $67.5 \mathrm{~cm}$ away from the inlet where the flow was relatively stable. Glass particles $0.145-0.175 \mathrm{~mm}$ in diameter were used in the 


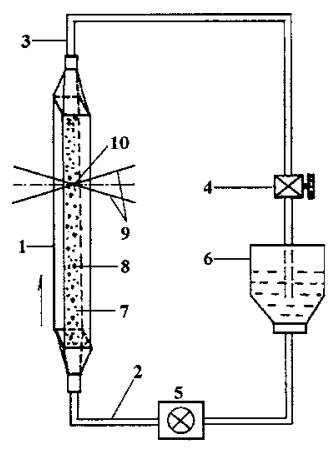

(a) Experimental setup

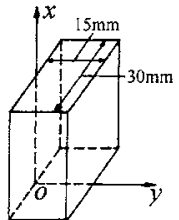

(b) Coordinate system
1. Experimental duct, 2. Up duct, 3. Down duct, 4. Control valve, 5. Pump, 6. Fluid

vessel, 7. Water flow, 8. Particle, 9. Laser beam, and 10. Measurement point

Fig. 1. Schematic view of experimental setup

experiments, and the maximum time-averaged velocity in the center of the duct was $0.65 \mathrm{~m} / \mathrm{s}$. The laser Doppler measurement technique was used to observe the number of particles passing through the observation point (the center point of the duct flow) during a certain time, which can be regarded as the number density of sediment particles or relative concentration. Experimental results of the transverse (perpendicular to the flow direction) velocity fluctuations of both phases and particle concentration along the centerline of the measurement section are shown in Figs. 2 and 3 , respectively.
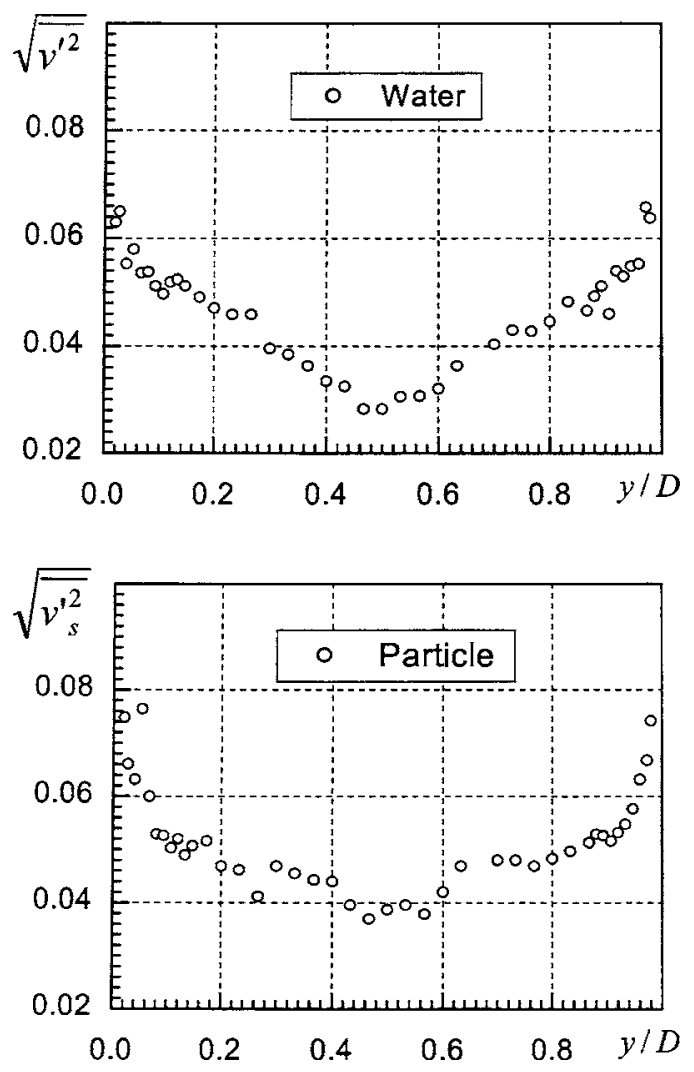

Fig. 2. Transverse (perpendicular to flow direction, i.e., $y$-direction) velocity fluctuations of both phases distributed along the transverse axis in the vertical rectangular duct flow. Here, $D$ is the width of rectangular duct, the unit of both $\sqrt{\overline{v^{\prime 2}}}$ and $\sqrt{\overline{v_{s}^{\prime 2}}}(\mathrm{~m} / \mathrm{s})$.

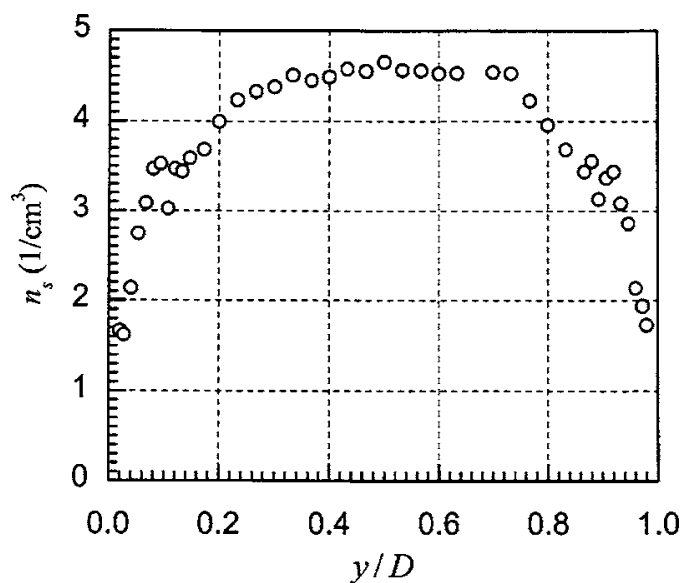

Fig. 3. Distribution of particle concentration (the number density of particles) along transverse axis in the vertical rectangular duct flow

Experimental results show that transverse velocity fluctuation profiles of both phases along the transverse axis are similar. The velocity fluctuations of both phases decrease first, reach a smallest value at the duct center, and then begin to increase. Comparing the velocity fluctuations of particles and water, the velocity fluctuation of the particle phase is generally larger than that of the liquid phase in the near-wall region. In addition, there exists a main difference between two phases: the turbulent flow velocity of the liquid phase decreases rapidly near the wall due to the influence of the wall, but this is not true for the particle phase, instead it increases near the wall. It is surmised that there are yet other particle fluctuation forms besides turbulent fluctuations caused by flow turbulence (Liu and Singh 2004). According to Liu and Lu (1993), the irregular pulsation caused by the collision between particles, or particle and wall, is a form of fluctuation which is different from turbulence. Consequently, the total fluctuation of particles can be regarded as a superposition of turbulent motion caused by flow turbulence and non-turbulent motion not caused by flow turbulence. On the contrary, the maximum value of the particle concentration occurs at the center of the duct and decreases gradually from the center to the walls.

Using the experimental results of particle velocity fluctuation and concentration, the profile of particle concentration in vertical flow given by Eq. (23) was validated, as illustrated in Fig. 4. The results of validation show that the value of $n_{s} \overline{v_{s}^{\prime 2}}$ is about a constant although there exist some perturbations. This comparison also indirectly verifies the validity of the present analysis of the vertical profile of sediment concentration distribution.

\section{Discussion on Sediment Diffusion in Water Flows}

Neglecting the flow uplift force in the vertical plane, Eq. (16) can be rewritten as

$$
\frac{\partial C}{\partial y}+\frac{C}{\overline{v_{s}^{\prime 2}}} \frac{\partial}{\partial y}\left(\overline{v_{s}^{\prime 2}}\right)=-\frac{C \omega}{\tau^{*} v_{s}^{\prime 2}}
$$

where $\tau^{*} \overline{v_{s}^{\prime 2}}$ factually expresses the diffusion coefficient of sediment, $\varepsilon_{s}$. Substitution of $\varepsilon_{s}$ for $\tau^{*} \overline{v_{s}^{\prime 2}}$ in Eq. (24) yields 


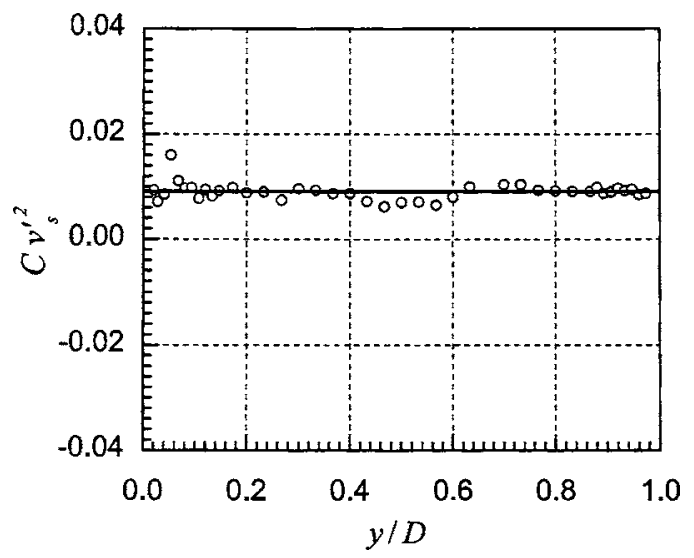

Fig. 4. Validation for Eq. (16) by experimental data (circles)

$$
\frac{\partial C}{\partial y}+\frac{C}{\overline{v_{s}^{\prime 2}}} \frac{\partial}{\partial y}\left(\overline{v_{s}^{\prime 2}}\right)=-\frac{C \omega}{\varepsilon_{s}}
$$

where the first term on the left-hand side of Eq. (25) represents the diffusion caused by the gradient of sediment concentration, and the second term on the left-hand side represents the diffusion caused by the gradient of particle's fluctuation intensity.

When $\overline{v_{s}^{\prime 2}}$ is constant (unchanged in the $y$-direction), Eq. (25) simplifies to

$$
\varepsilon_{s} \frac{\partial C}{\partial y}=-C \omega
$$

Eq. (26) is in complete accord with the form yielded by the diffusion theory. This confirms that the diffusion theory, based on Fick's law, is only valid for $\overline{v_{s}^{\prime 2}}$ being constant, i.e., the intensity of vertical fluctuation of particles is uniform in the vertical plane. That is to say, the diffusion theory neglects the sediment diffusion caused by the gradient of turbulent intensity. In fact, some researchers have explained this kind of diffusion, and defined it as dispersion (Crowe et al. 1988; Graham and James 1996). For shear flow, the variation of turbulent intensity is pronounced, and therefore the sediment diffusion caused by the gradient of the particle fluctuation intensity must be accounted for sediment transport.

The present analysis shows that the suspension and diffusion of particles depend on the particle fluctuation. Generally, in the mainstream region the particle fluctuation is mainly caused by flow turbulence, and the difference between particle fluctuation and flow turbulent intensities is relatively small. Thus, the particle fluctuation can be completely replaced with flow turbulence. However, the particle phase has other fluctuation forms that are different from flow turbulence, especially in the thin region near the bed, which can cause a rather significant difference between particle fluctuation and flow turbulence intensities.

Experimental data in open channel flow have demonstrated that the turbulence intensity in the mainstream region changes little and is approximately uniform. This means that the traditional diffusion theory can still be applied as a good approximation. But in the region near the bed, the traditional diffusion theory must be revised because of greater variation of turbulence intensity. For instance, in vertical pipe flow, the particle diffusion caused by the gradient of turbulence intensity is strong and it can balance the particle diffusion caused by the gradient of particle concentration. This also indicates that the diffusion caused by the gradient of turbulence intensity can be rather strong under certain

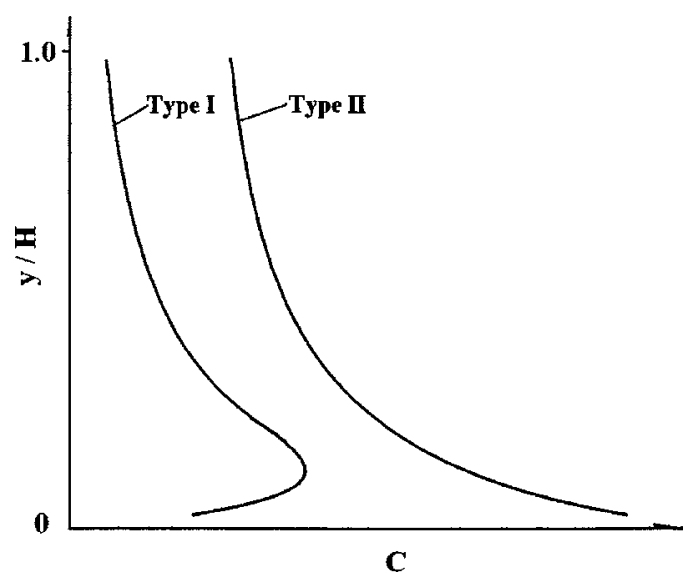

Fig. 5. Two types of sediment concentration profile

conditions. The way to modify the diffusion theory is to add the diffusion caused by the gradient of turbulence intensity. In this manner, many previous research results based on the diffusion theory can be employed in practical applications. Therefore, it is essential to divide the whole flow region into two parts: the mainstream region where the diffusion theory is still applicable, and the region near the bed where the diffusion theory needs to be revised.

\section{Discussion of Two Patterns of Vertical Profiles of Sediment Concentration}

The traditional diffusion theory yields that in open channel flow the sediment concentration always gradually decreases from bed to the water surface. That is why for many years the vertical profile of suspended concentration was described in only one way: "up small and down great." However, many observations in flume flows, pipe flows and natural river flows show that sediment concentration may increase with height up to the boundary of the thin region near the bed in many situations. In other words, sediment concentration increases at first, then begins to decrease when the height from the bed reaches a critical value. This phenomenon cannot be explained by the diffusion theory, and therefore it is commonly thought to be an incorrect result caused by the measurement error.

With the remarkable development of the two-phase flow theory and measurement techniques, considerable progress has been achieved in the understanding of the sediment concentration distribution in the vertical plane. Ni and Wang (1987) pointed out that there indeed exist two patterns of sediment concentration distribution profiles (see Fig. 5), and analyzed the reasons leading to these two kinds of profiles. They regarded that the greater uplift force near the bed is the main reason leading to the sediment concentration increase from the bed to a small height. Also, they reported that the interaction of particles and bed may affect the profile of sediment concentration, but did not provide any further analysis. Nevertheless, their investigation partly remedied the shortcoming of the diffusion theory.

From the results of the present study, this problem can be analyzed and explained easily. Changing the form of Eq. (16) yields 


$$
\left(\overline{v_{s}^{\prime 2}}\right) \frac{\partial C}{\partial y}+C \frac{\partial}{\partial y}\left(\overline{v_{s}^{\prime 2}}\right)=-\left(\frac{\omega}{\tau^{*}}-a\right) C
$$

One further obtains

$$
\frac{\partial}{\partial y}(\ln C)=-\left[\frac{\partial}{\partial y}\left(\overline{v_{s}^{\prime 2}}\right)+\frac{\omega}{\tau^{*}}-a\right] / \overline{v_{s}^{\prime 2}}
$$

Eq. (28) shows that so long as $\left(\partial\left(\overline{v_{s}^{\prime 2}}\right) / \partial y+\omega / \tau^{*}-a\right)$ is less than zero, $\partial(\ln C) / \partial y$ will be greater than zero, namely, the sediment concentration will increase with height. For open channel flows, the term $\omega / \tau^{*}$ is always greater than zero, the term $(a)$ is also greater than zero, but the term $\partial\left(\overline{v_{s}^{\prime 2}}\right) / \partial y$ is generally less than zero. Commonly, the term $\omega / \tau^{*}$ is rather large as compared with $\left|\partial\left(\overline{v_{s}^{\prime 2}}\right) / \partial y\right|+a$, especially in the mainstream region where both $\left|\partial\left(\overline{v_{s}^{\prime 2}}\right) / \partial y\right|$ and $(a)$ are generally very small. Therefore, in most situations, the value of $\left(\partial\left(\overline{v_{s}^{\prime 2}}\right) / \partial y+\omega / \tau^{*}-a\right)$ is less than zero, which results in the type $I$ profile of the concentration distribution.

However, in many situations, $\left|\partial\left(\overline{v_{s}^{\prime 2}}\right) / \partial y\right|$ and the flow uplift (a) are great enough in the region near the bed, which may make the following equation correct in a small layer near the bed

$$
\left|\frac{\partial}{\partial y}\left(\overline{v_{s}^{\prime 2}}\right)-a\right|>\frac{\omega}{\tau^{*}}
$$

Or

$$
\frac{\partial}{\partial y}\left(\overline{v_{s}^{\prime 2}}\right)+\frac{\omega}{\tau^{*}}-a<0
$$

In this situation, the sediment concentration will increase with height from the bed in a thin region, and exhibit Type II profile.

From the experimental observations employed in this study, the fluctuation intensity of particles changes rapidly near the wall. Experimental data obtained in horizontal flows also show that the fluctuation intensity of particles decreases rapidly with height in the closed bed, which commonly leads to a greater value of $\left|\partial \overline{v_{s}^{\prime 2}} / \partial y\right|$. This then leads to a reverse distribution of sediment concentration near the bed.

The above analysis shows that both the gradient of vertical fluctuation intensity of particles and the flow uplift force can cause the reverse distribution of sediment concentration. In general, the flow uplift force is not sufficiently large. It is therefore hypothesized that the gradient of vertical fluctuation intensity of particles is a more significant factor leading to Type II profile of concentration, as compared with the flow uplift force. Of course, the flow uplift force may be also strong under some specific situations. In other words, both the gradient of vertical fluctuation intensity of particles and the flow uplift force can cause Type II profile of sediment concentration distribution.

\section{Conclusions}

The following conclusions are drawn from this study:

1. The gravity and buoyancy forces acting on sediment particles can be balanced by the momentum of fluctuations of moving particles, the uplift force and the flow resistance that the water flow exerts on the particles. The momentum transport caused by the vertical fluctuation of particles is the most significant factor leading to sediment particle suspension in water flow. The steady vertical distribution of sediment concentration mainly depends on the vertical fluctuation of particles and its distribution.

2. Based on the momentum equilibrium of group particle motion in the vertical plane, a formula expressing the profile of sediment concentration distribution is derived, which demonstrates that besides the gradient of concentration the gradient of fluctuation intensity of particles can lead to the diffusion of particles. The traditional diffusion theory based on Fick's law neglects the sediment diffusion caused by the gradient of turbulent intensity, and is only applicable for uniform turbulent flow.

3. Both the gradient of vertical fluctuation intensity of particles and the flow uplift force may cause the reverse distribution of sediment concentration. The rapid decrease of the gradient of particle fluctuation intensity with height from the bed and the flow uplift force in the region near the bed are the key factors leading to Type II profile of sediment concentration.

\section{Acknowledgments}

This research was financially supported by the Key Projects of the Chinese Natural Science Foundation (No. 10332050).

\section{Notation}

The following symbols are used in this paper:

$a=$ acceleration velocity due to by the uplift force $F_{L}$;

$C=$ sediment concentration;

$D=$ width of rectangle duct;

$F_{y}=$ uplift force of flow acting on sediment particles;

$f_{L}=$ laminar flow resistance;

$f_{T}=$ turbulent flow resistance;

$f_{y}=$ vertical resistance of the sediment particles to water flow;

$g=$ gravitational acceleration;

$g_{y}=$ gravitational acceleration exponent in the $y$-direction;

$J=$ slope gradient $(J=\tan \theta \approx \sin \theta)$;

$K=$ constant;

$k=$ constant;

$m_{s}=$ mass of sediment particle;

$n_{s}=$ number density of sediment particles;

$p=$ pressure of water flow;

$U_{s}^{T}=$ turbulent diffusion velocity of particle;

$u_{s}=$ particle velocity in the horizontal (or longitudinal) direction;

$u_{s}^{\prime}=$ particles velocity fluctuation in the horizontal (or longitudinal) direction;

$v_{s}=$ particle velocity in the vertical (or transverse) direction;

$v^{\prime}=$ turbulent velocity of flow in the vertical (or transverse) direction;

$v_{s}^{\prime}=$ particles velocity fluctuation in the vertical (or transverse) direction;

$x=$ coordinate along in the horizontal (or longitudinal) direction;

$y=$ coordinate along in the vertical (or transverse) direction;

$\alpha=$ fractional number of fluid by volume;

$\alpha_{s}=$ fractional number of sediment particle by volume; 
$\Delta U_{y}^{T}=$ difference of diffusion velocity of two phases;

$\varepsilon_{s}=$ sediment diffusion coefficient;

$\theta=$ slope angle of slope;

$\rho=$ density of fluid;

$\rho_{s}=$ density of sediment particles;

$\sigma=$ fractional density of fluid;

$\sigma_{s}=$ fractional density of sediment particles;

$\tau^{*}=$ relaxation time of sediment particles; and

$\omega=$ deposition velocity of sediment particles.

\section{References}

Aísa, L., Garcia, J. A., Cerecedo, L. M., Palacín, I. G., and Calvo, E. (2002). "Particle concentration and local mass flux measurements in two-phase flows with PDA. Application to a study on the dispersion of spherical particles in a turbulent air jet." Int. J. Multiphase Flow, 28(2), 301-324.

Batchelor, G. K. (1965). "The motion of small particles in turbulent flow." 2nd Australasian Conf. on Hydraulics and Fluid Mechanics, Univ. Auckland, Auckland, New Zealand.

Chien, N., and Wan, Z. (1999). Mechanics of sediment transport, J. S. McNown, translator, ASCE, Reston, Va., 63-113.

Crowe, C. T., Chung, J. N., and Troutt, T. R. (1988). "Particle mixing in free shear flows." Prog. Energy Combust. Sci., 14(3), 171-194.

Derevich, I. V. (2000). "Statistical modeling of mass transport in turbulent two-phase dispersed flows. I: Model development." Int. J. Heat Mass Transfer, 43(19), 3709-3723.

Dyer, K. R., and Soulsby, R. L. (1988). "Sand transport on the continental shelf." Annu. Rev. Fluid Mech., 20, 295-324.

Fu, X. D., and Wang, G. Q. (2003). "Kinetic model of particulate phase in dilute solid-liquid two-phase flows." Acta Mech. Sin., 35(6), 650-659 (in Chinese).

Fu, X. D., Wang, G. Q., and Dong, Z. N. (2001). "Theoretical analysis and numerical computation of dilute solid/liquid two-phase pipe flow." Sci. China, Ser. E: Technol. Sci., 44(3), 298-308.

Graham, D. I., and James, P. W. (1996). "Dispersion of solid particles in turbulent flow through pipe expansions." J. Manage. Math., 7(2), 149-179.

Hyland, K. E., McKee, S., and Reeks, M. W. (1999). "Derivation of a pdf kinetic equation for the transport of particles in turbulent flows." J. Phys. A: Math Gen., 32(34), 6169-6190.

Ismail, H. M. (1952). "Turbulent transfer mechanics of turbulence." Trans. Am. Soc. Civ. Eng., 117, 409-446.

Itakura, T., and Kishi, T. (1980). "Open channel flow with suspended sediments." J. Hydr. Div., 106(8), 1325-1343.

Kaushal, D. K., Seshadri, N., and Singh, S. N. (2002). "Prediction of concentration and particle size distribution in the flow of multi-sized particulate slurry through rectangular duct." Appl. Math. Model., 26(10), 941-952.

Liu, D. Y. (1993). Fluid dynamics of two-phase systems, Chinese Higher Education Press, Beijing (in Chinese).

Liu, D. Y., and Lu, Z. M. (1993). "Various kinds of random motion and stress in two-phase flows." Proc., 2nd Int. Conf. on the Fluid Mechanics, Peking Univ. Press, Beijing, 782-788.
Liu, Q. Q. (1998). "LDV measurements and experimental study of water and sediment two-phase flow." J. Sediment Res. 1998, 2, 71-79 (in Chinese).

Liu, Q. Q., and Singh, V. P. (2004). "Fluid-solid interaction in particleladen flows." J. Eng. Mech., 130(12), 1476-1485.

Lu, Z. M., Li, G. D., and Peng, W. S. (1988). "LDA measurements of bubble-water two-phase flow." Acta Mech. Sin., 20(6), 489-495 (in Chinese).

McLean, S. R. (1992). "On the calculation of suspended load for noncohesive sediments.” J. Geophys. Res., 97(C4), 5759-5770.

Montes, J. S., and Ippen, A. T. (1973). "Interaction of two-dimensional turbulent flow with suspended particles." Rep. No. 164, Lab. Water Resources and Hydrodynamics, Massachusetts Inst. Tech., Cambridge, Mass.

Ni, J. R., and Wang, G. Q. (1987). "Studies on the two patterns of vertical distribution of particle concentrations and their formation cause." J. Hydraul. Eng., 17(7), 60-67 (in Chinese).

Ni, J. R., and Wang, G. Q. (1991a). "On the vertical distribution of suspended sediment concentration." Int. J. Sediment Res., 6(3), 5572.

Ni, J. R., and Wang, G. Q. (1991b). "Vertical sediment distribution." J. Hydraul. Eng., 117(9), 1184-1194.

Nielsen, P. (1991). "Combined convection and diffusion: A new framework for suspended sediment modeling." Proc., Coastal Sediments '91, Seattle, ASCE, 1, 418-431.

Nielsen, P. (1995). "Suspended sediment concentration profiles." Appl. Mech. Rev., 38(9), 564-569.

Nino, Y., and Garcia, M. H. (1996). "Experiments on particle-turbulence interactions in the near-wall region of open channel flow: Implications for sediment transport." J. Fluid Mech., 326, 285-319.

O'Brien, M. P. (1933). "Review of the theory of turbulent flow and its relations to sediment transportation." Trans. Amer. Geophys. Union, 14, 487-491.

Rouse, H. (1937). "Modern conceptions of the mechanics of turbulence." Trans. Am. Soc. Civ. Eng., 102, 463-505.

Sommerfeld, M., and Huber, H. (1999). "Experimental analysis and modeling of particle-wall collisions." Int. J. Multiphase Flow, 25(6-7), 1457-1489.

Tsuji, Y., and Morikawa, Y. (1982). "LDV measurements of an air-solid two-phase flow in a horizontal pipe." J. Fluid Mech., 120, 385-409.

van Rijn, L. C. (1984). "Sediment transport. Part II: Suspended load transport." J. Hydraul. Eng., 110(11), 1613-1641.

Vanoni, V. A. (1946). "Transport of suspended sediment by water." Trans. Am. Soc. Civ. Eng., 111, 67-102.

Wang, G., and Ni, J. (1990). "Kinetic theory for particle concentration distribution in two-phase flow." J. Eng. Mech., 116(12), 2738-2748.

Wang, G. Q., and Ni, J. R. (1991). "The kinetic theory for dilute solid/ liquid two-phase flow." Int. J. Multiphase Flow, 17(2), 273-281.

$\mathrm{Xu}$, Y., and Zhou, L. X. (2000). "A second order moment two-phase turbulence model based on the Lagrangian PDF." J. Comput. Phys., 17(6), 633-6401 (in Chinese).

Zaichik, L. I., et al. (1997). "Modeling of dynamics, heat transfer, and combustion in two-phase turbulent flows. I: Isothermal flows." Exp. Therm. Fluid Sci., 15(4), 291-310.

Zhou, D., and Ni, J. R. (1995). "Effects of dynamic interaction on sediment-laden turbulent flows," J. Geophys. Res., 100(C1), 981-996. 